\title{
Value of transoesophageal echocardiography in surgical ligation of coronary artery fistulas
}

\author{
I D Cox, S C Heald, A J Murday
}

A 62 year old man was referred to hospital as an emergency with a three week history of worsening dyspnoea and orthopnoea. His general practitioner had previously noted a late systolic murmur, and a transthoracic echocardiogram performed seven months previously had shown prolapse of the posterior mitral valve leaflet with moderate mitral regurgitation.

Examination on admission showed a regular tachycardia of 150 beats per minute with a systemic blood pressure of $140 / 90 \mathrm{~mm} \mathrm{Hg}$. His apex beat was hyperdynamic and displaced laterally toward the anterior axillary line. At auscultation a loud, apical, pansystolic murmur with an associated thrill was heard. There were fine crepitations at both lung bases but no signs of right ventricular failure. An electrocardiogram revealed atrial flutter with 2 to 1 atrioventricular block, and on chest $x$ ray there was cardiomegaly and evidence of pulmonary venous congestion. The patient was treated with intravenous frusemide to good effect and the atrial flutter, which did not respond to initial treatment with intravenous amiodarone, was successfully DC cardioverted to sinus rhythm under general anaesthesia.

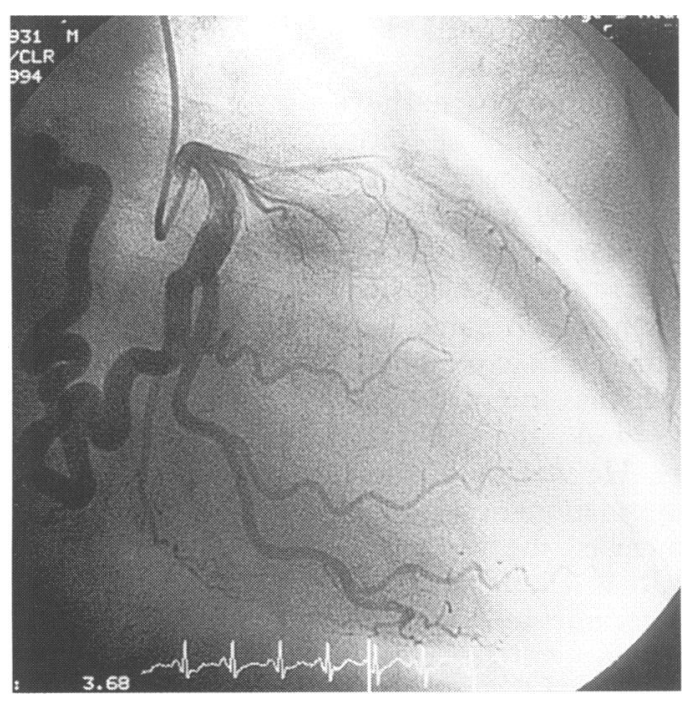

Figure 1 Selective coronary angiogram taken from the right anterior oblique projection showing a large, tortuous coronary artery fistula originating from the circumflex artery and terminating in the superior vena cava at its junction with the right atrium.
Transthoracic echocardiography showed severe mitral regurgitation with evidence of acute chordal rupture of the mitral valve. Subsequent cardiac catheterisation confirmed grade IV mitral regurgitation but selective coronary angiography also showed a large, tortuous coronary artery fistula running between the left circumflex artery and the superior vena cava (fig 1). An oxygen saturation run showed a small saturation jump at the level of the high right atrium, indicating a left to right heart shunt $(\mathrm{Qp} / \mathrm{Qs} 1 \cdot 2: 1)$ at that level. The coronary arteries were otherwise normal. Left ventricular end diastolic pressure was $18 \mathrm{~mm}$ $\mathrm{Hg}$, pulmonary artery pressure was $42 / 20 \mathrm{~mm}$ $\mathrm{Hg}$, and mean pulmonary artery wedge pressure was $17 \mathrm{~mm} \mathrm{Hg}$ (v wave of $30 \mathrm{~mm}$ $\mathrm{Hg}$ ).

The patient was referred for surgery and operative findings confirmed the diagnosis of chordal rupture of the posterior mitral valve leaflet. The valve was repaired using a quadrangular resection of the posterior leaflet, apposition of the leaflet edges, and an annuloplasty with a size 34 . Carpentier ring. Examination of the fistula showed a large vessel arising from the circumflex system, traversing across the roof of the left atrium, and entering the superior vena cava at its junction with the right atrium. The fistula was initially ligated securely and divided at its distal junction with the superior vena cava. Perioperative transoesophageal echocardiography (TOE) confirmed a satisfactory mitral valve repair but also showed persistent flow through the fistula (fig 2). Further proximal ligation of the fistula was performed closer to the circumflex system after which TOE confirmed that flow in the fistula had been abolished. Postoperatively the patient made an uncomplicated recovery.

Coronary artery fistulas are rare, with an incidence of $0.2 \%$ in one large angiographic series. ${ }^{1}$ Such fistulas arise in about equal proportions from the right and left coronary systems $^{1}$ and drain into the low pressure venous system, most commonly the right ventricle or right atrium, in over $90 \%$ of cases. Preoperative identification of coronary fistulas in patients undergoing mitral valve surgery is important as it may have technical implications regarding the surgical approach to the valve. It would also be regrettable to miss the opportunity to ligate a fistula during surgery
Hospital Medical School, Cranmer Terrace, Tooting,

Accepted for publication 13 March 1996 


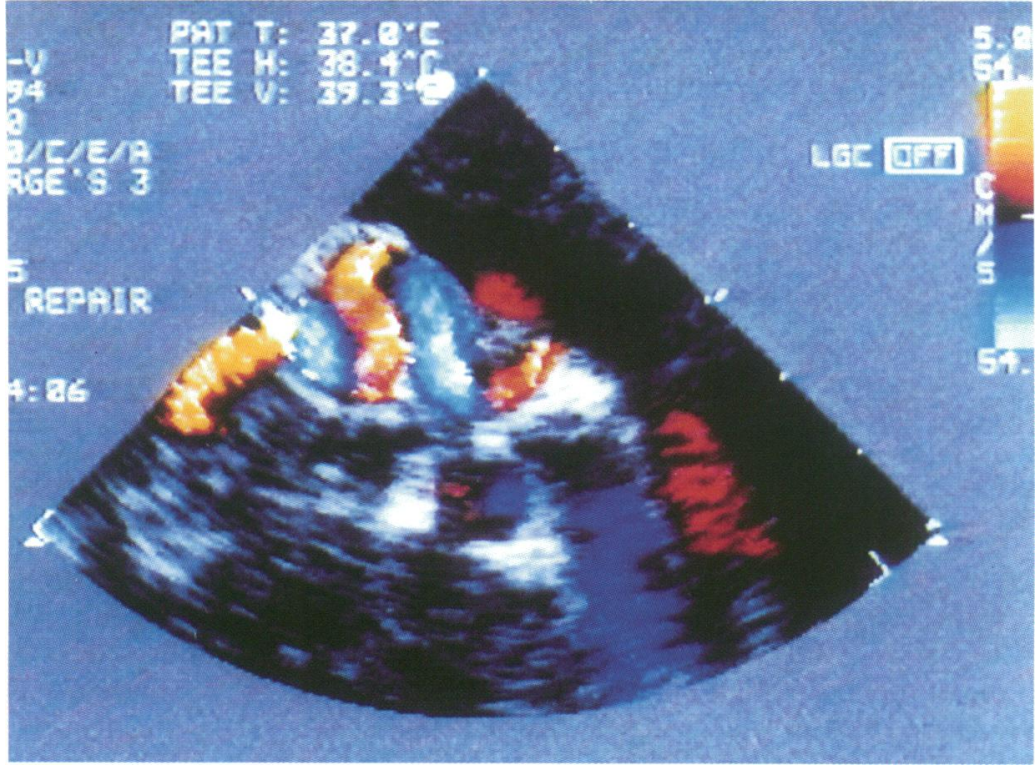

Figure 2 Perioperative transoesophageal echocardiogram of the tortuous coronary fistula. Colour flow Doppler demonstrates the presence of residual flow in the fistula despite initial distal surgical ligation. coronary fistulas is preferentially performed at their distal, low pressure end because this reduces the risk of compromising flow in the feeding artery. However, coronary fistulas often terminate in more than one distal connection and successful distal ligation can prove difficult. This case supports the value of TOE for the perioperative evaluation of coronary fistulas $^{4-6}$ and illustrates how this technique may be used to identify cases that require proximal ligation.

1 Hobbs RE, Millit HD, Raghavan PV, Moodie DS, Sheldon WC. Coronary artery fistulae: a 10-year review. Cleveland Clinic Quarterly 1982;49:191-7.

2 Wilde P, Watt I. Congenital coronary artery fistulae: six new cases with a collective review. Clin Radiol 1980;31: 301-11.

3 Liberthson RR, Sagar K, Berkoben JP, Weintraub RM, Levine FH. Congenital coronary arteriovenous fistula. Report of 13 patients, review of the literature and delineation of management. Circulation 1979;59:849-54.

4 Giannoccaro PJ, Sochowski RA, Morton BC, Chan KI Complementary role of transoesophageal echocardiography to coronary angiography in the assessment of phy to coronary angiography in the assessment

5 Calafiore PA Raymond R Schiavone WA Rosenkranz ER. Precise evaluation of a complex coronary arterioER. Precise evaluation of a complex coronary arteriovenous fistula: the utility of transoesophageal

because, although they are often asymptomatic, such fistulas may lead to significant late complications. ${ }^{23}$ Initial surgical ligation of
6 Stevenson JG, Sorensen GK, Stamm SJ, McCloskey JP, Hall DG, Rittenhouse EA. Intraoperative transAnn Thorac Surg 1994;57:1217-21. esophageal echocardiography of coronary artery fistulas.

\section{STAMPS IN CARDIOLOGY}

\section{Paul Dudley White (1886-1973)}

This 3 cent American stamp was issued on 15 September 1986 at a ceremony held during, and as part of, the Tenth World Congress of Cardiology held in Washington DC. It forms part of the Great Americans series.

Paul Dudley White was born in Roxbury, Massachusetts, and graduated from Harvard Medical School. He went to London in 1913 to study the new science of electrocardiography under Dr (later Sir) Thomas Lewis, and was then appointed to the staff of the Massachusetts General Hospital where he founded the Heart Clinic. His 931 page Heart Disease written in 1931 became a classic textbook. He was founder member in 1922 of the American Heart Association and became its president in 1942. Together with colleagues from abroad he founded the International Society of Cardiology and the World Congresses of Cardiology. He was tireless in promoting the cause of international cooperation in cardiology and travelled the world to promote this aim, especially to China and the Soviet Union. In 1955 he became more widely known to the public when he was the chief consultant to President Dwight D Eisenhower who had a myocardial infarction. White had a life-long interest in the prevention of heart disease, especially coronary heart disease, and was a strong advocate of measures such as

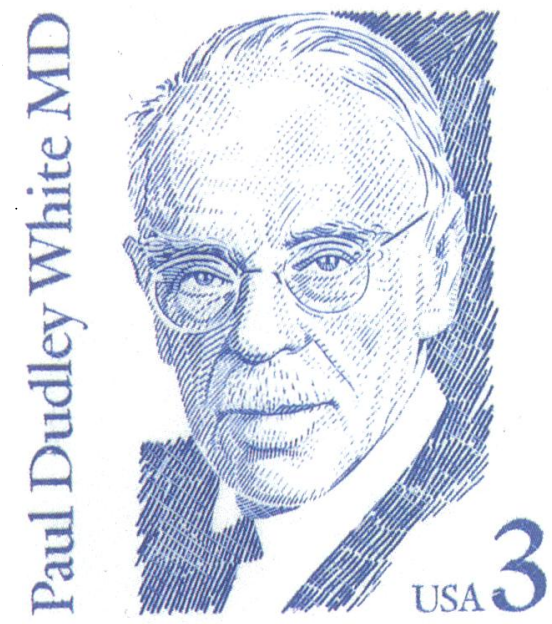

diet, weight control, and exercise. A wellknown photograph showed him and his wife riding their bicycles. His pioneer work in electrocardiography included the first published description of the features of pulmonary embolism, and he is remembered as co-author of the early definitive paper on the WolffParkinson-White syndrome.

M K DAVIES A HOLLMAN 